\title{
Minireview
}

\section{The Pharmacological Case for Cannabigerol}

\author{
Rahul Nachnani, Wesley M. Raup-Konsavage, and @Kent E. Vrana \\ Department of Pharmacology, Penn State College of Medicine, Hershey, Pennsylvania \\ Received September 15, 2020; accepted November 4, 2020
}

\begin{abstract}
Medical cannabis and individual cannabinoids, such as $\Delta 9$ tetrahydrocannabinol $(\Delta 9-\mathrm{THC})$ and cannabidiol $(\mathrm{CBD})$, are receiving growing attention in both the media and the scientific literature. The Cannabis plant, however, produces over 100 different cannabinoids, and cannabigerol (CBG) serves as the precursor molecule for the most abundant phytocannabinoids. CBG exhibits affinity and activity characteristics between $\triangle 9$-THC and CBD at the cannabinoid receptors but appears to be unique in its interactions with $\alpha$-2 adrenoceptors and 5-hydroxytryptamine (5- $\left.\mathrm{HT}_{1 \mathrm{~A}}\right)$. Studies indicate that $\mathrm{CBG}$ may have therapeutic potential in treating neurologic disorders (e.g., Huntington disease, Parkinson disease, and multiple sclerosis) and inflammatory bowel disease, as well as having
\end{abstract}

antibacterial activity. There is growing interest in the commercial use of this unregulated phytocannabinoid. This review focuses on the unique pharmacology of CBG, our current knowledge of its possible therapeutic utility, and its potential toxicological hazards.

\section{SIGNIFICANCE STATEMENT}

Cannabigerol is currently being marketed as a dietary supplement and, as with cannabidiol (CBD) before, many claims are being made about its benefits. Unlike CBD, however, little research has been performed on this unregulated molecule, and much of what is known warrants further investigation to identify potential areas of therapeutic uses and hazards.

\section{Introduction}

Cannabinoids as Medicine. The use of Cannabis sat$i v a$ as a medicine dates back millennia. In ancient China, marijuana was used to treat gout, malaria, digestive disorders, and menstrual pain (Bostwick, 2012; Russo, 2016; Kinghorn et al., 2017; Ryz et al., 2017; Baron, 2018; Ambrose and Simmons, 2019). Cannabis was introduced to Western medicine by William O'Shaugnessy, who proposed its use for the treatment of rheumatism and seizures (Bostwick, 2012; Russo, 2016; Kinghorn et al., 2017; Baron, 2018). However, the use of medicinal marijuana fell out of favor toward the end of the 19th century and continued to decline until it was banned by the Controlled Substances Act of 1970 (Bostwick, 2012; Sacco, 2014; Kinghorn et al., 2017). Since that time, few cannabinoid drugs have been approved for human use. These drugs include nabilone in 1985, dronabinol in 1986, rimonabant in 2006 (in Europe; withdrawn in 2008), Sativex (in

This work was supported by a sponsored research agreement from Pennsylvania Options for Wellness (to K.E.V.).

No author has an actual or perceived conflict of interest with the contents of this article.

https://doi.org/10.1124/jpet.120.000340.
Europe) in 2010, and Epidiolex in 2018. A growing number of countries have also approved the use of medical marijuana for treating a variety of medical conditions; however, the data that support the use of marijuana for treatment are often anecdotal or from small studies.

Cannabigerol and Cannabinoid Synthesis. Cannabinoid synthesis begins with the precursor molecules olivetolic acid and geranyl-pyrophosphate, which combine to form cannabigerolic acid (CBGA) (Shoyama et al., 1975; Fellermeier and Zenk, 1998; Fellermeier et al., 2001; Gülck and Møller, 2020). CBGA serves as the precursor to most other cannabinoids and is converted to $\Delta 9$-tetrahydrocannabinolic acid ( $\triangle 9$-THCA), cannabidiolic acid (CBDA), and cannabichromenic acid (Fig. 1). Because CBGA serves as the precursory molecule to the other cannabinoids, it is normally found in very low quantities in Cannabis; however, strains with reduced activity of the three major synthesis enzymes can accumulate higher levels of CBGA (Fellermeier and Zenk, 1998; Fellermeier et al., 2001). All enzymatically produced cannabinoids (including CBG) are produced as their acidic form and are then decarboxylated by heat to create the "active" form.

With the recent deregulation of cannabidiol (CBD) and other hemp-derived cannabinoids, such as CBG, cannabichromene

ABBREVIATIONS: ADHD, attention-deficit hyperactivity disorder; $\mathrm{CB} 1$, cannabinoid receptor 1; CB2, cannabinoid receptor 2; CBC, cannabichromene; CBD, cannabidiol; CBDA, cannabidiolic acid; CBG, cannabigerol; CBGA, cannabigerolic acid; GPCR, G protein-coupled receptor; GPR55, G protein-coupled receptor 55; HD, Huntington disease; 5-HT, 5-hydroxytryptamine; IL, interleukin; PFC, prefrontal cortex; PPAR, peroxisome proliferator-activated receptor; $\triangle 9$-THC, $\Delta$ 9-tetrahydrocannabidiol; $\Delta 9$-THCA, $\Delta 9$-tetrahydrocannabidiolic acid; TRPM, transient receptor potential cation channel subfamily M; TRPV, transient receptor potential cation channel subfamily V. 


\section{Geranyl Pyrophosphate Olivetolic Acid}

Geranyl-pyrophosphate-olivetolic acid Geranyltransferase<smiles>CCCCCc1cc(O)c(C/C=C(\C)CCC=C(C)C)c(O)c1C(=O)O</smiles>

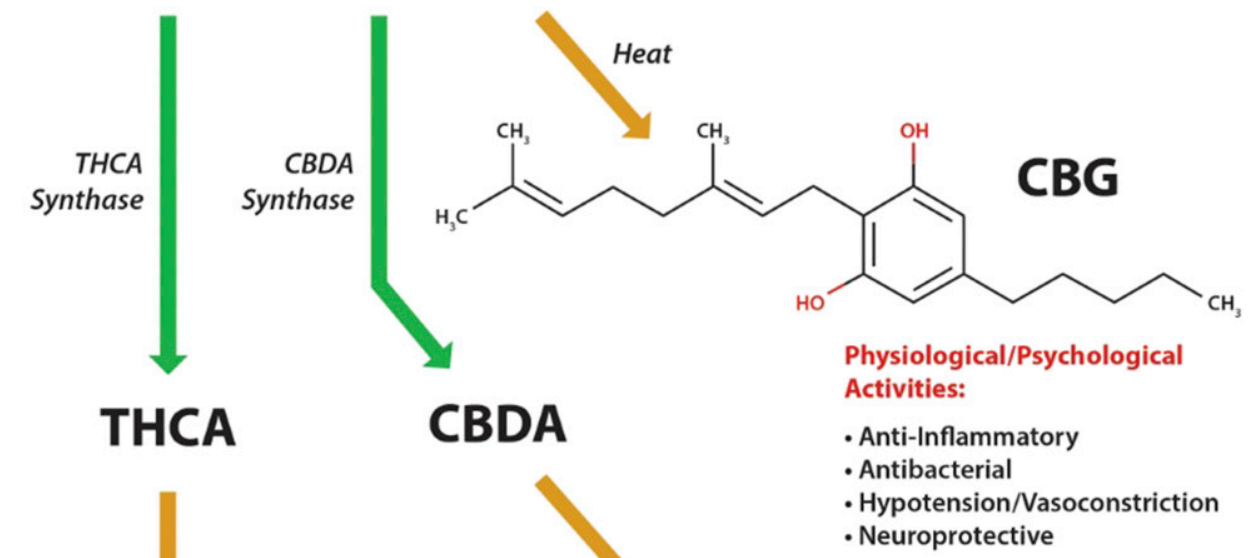<smiles>CCCCCc1cc(O)c2c(c1)OC(C)(C)[C@@]1(C)CCC(C)=C[C@H]21</smiles>

Physiological/Psychological Activities:

- Euphorigenic - Appetite Stimulant - Antiemetic - Analgesic
Heat<smiles>C=C(C)[C@H]1CCC(C)=C[C@H]1c1c(O)cc(CCCCC)cc1O</smiles>

Physiological/Psychological Activities: - Anticonvulsant
-Anti-Inflammatory • Analgesic
Fig. 1. Biosynthesis pathway of the primary cannabinoids. Cannabinoid biosynthesis begins with the combination of geranyl pyrophosphate and olivetolic acid to form CBGA. CBGA serves as the substrate for the synthesis of $\triangle 9$-THCA and CBDA. Decarboxylation of CBGA, $\triangle 9$-THCA, and CBDA by heat results in CBG, $\triangle 9$-THC, and CBD, respectively. Because CBGA serves as the substrate for the synthesis of the major cannabinoids, very little is typically found in material from Cannabis sp.
(CBC), and cannabinol (2018 Farm Bill), there is growing interest in cannabinoid pharmacology (https://www.usda.gov/farmbill). For instance, in spite of having CBG as a common precursor, $\Delta 9$ THC, CBD, and CBC have dramatically different physiologic effects. In the case of the two most widely studied compounds, $\Delta 9$ THC is known to produce euphoria and appetite stimulation (Volkow et al., 2014), and CBD is noneuphorigenic and is thought to be antiepileptic (Jones et al., 2010) and anti-inflammatory (Carrier et al., 2006). Much less is known about CBG and CBC, but since there are differences in ring structure, it is not surprising that they have differing pharmacological properties; however, cannabinoids other than $\triangle 9$-THC and CBD have had little exploration and characterization.

Interest in Alternative Cannabinoids. Recent events are prompting increased interest in the pharmacotherapeutic potential of the $100+$ cannabinoid compounds. First, there exists growing belief that the beneficial effects of Cannabis derive from the entourage effect: all of the Cannabis-derived cannabinoids, terpenoids, and flavonoids acting in concert. Although there is little evidence to document this, it does support the notion that the entire plant material needs to be consumed. Then, with the explosion in sales of CBD products, entrepreneurs are searching for the next economic market, and it appears that CBG oil may prove to be that market. Indeed, several commercial outlets are advertising CBG as the "mother of all cannabinoids"-presumably because in Cannabis it is a common precursor. However, CBG will not give rise to the myriad other cannabinoids when taken into the human body because of the lack of necessary conversion pathways.

Work in recent years has garnered interest in other cannabinoids, such as $\mathrm{CBC}$ and $\Delta 9$-tetrahydrocannabivarin, but more studies are needed. $\Delta 9$-Tetrahydrocannabivarin is suggested to induce similar effects as $\Delta 9$-THC without excessive appetite stimulation (Tudge et al., 2014), and studies of CBC show it can improve inflammation-related pain (Wirth et al., 1980). 
In the present review, we explore potential therapeutic benefits and adverse side effects of CBG. Recent literature on CBG has revealed that its pharmacology addresses therapeutic targets distinct from those of $\triangle 9-\mathrm{THC}$ and CBD. We summarize previous investigations into therapeutic applications and propose new areas of interest for CBG's medical use. In vitro and in vivo studies suggest a potential future for CBG to address unmet needs in medical therapy, including its actions on adrenergic, serotonergic, peroxisome proliferatoractivated receptor (PPAR), and cannabinoid receptor families (Cascio et al., 2010; Rock et al., 2011; O'Sullivan, 2016; Navarro et al., 2018).

\section{Discussion}

\section{The Unique Pharmacologic Properties of CBG}

CBG is distinct from $\triangle 9$-THC and CBD in its pharmacological profile (as summarized in Tables 1 and 2). In a variety of different ways, CBG seems to reside, pharmacologically, in between $\triangle 9$-THC and CBD. From the receptor binding data presented in Table $1, \mathrm{CBG}$ is more like $\triangle 9$-THC at the CB1/CB2 receptors than CBD, but with a lower affinity (by a factor of between 5-fold and 27-fold) (Pertwee, 2008; Cascio et al., 2010; Pollastro et al., 2011; Rosenthaler et al., 2014; Navarro et al., 2018, 2020). However, a 2012 study of CBG in human cell culture demonstrated negligible binding affinities for CBG at $\mathrm{CB} 1$ and $\mathrm{CB} 2$ receptors (Granja et al., 2012). More research is required to better understand the effects of CBG in vivo on cannabinoid receptor function and activity. In addition, CBD and CBG are very comparable at six transient receptor potential cation channels (TRPA1, TRPV1, TRPV2. TRPV3, TRPV4, and TRPM8) with relatively minor differences in affinity (generally less than 5 -fold differences) (De Petrocellis et al., 2011, 2012; Pollastro et al., 2011; Muller et al., 2019). Important differences or gaps in our knowledge, however, exist for three key players. First, for GPR55 (the potential nonhomologous CB3 receptor), there is no information on CBG binding (Ryberg et al., 2007). Second, CBG appears to be a very potent (nanomolar to sub-nanomolar affinity) agonist at the $\alpha$-2 adrenoceptor (Cascio et al., 2010). Physiologically, this is so potentially important that it is the subject of extensive discussion below. At the present time, there are no data on this receptor for CBD and $\triangle 9$-THC. Finally, there is a clear differentiation of CBG and CBD at the $5-\mathrm{HT}_{1 \mathrm{~A}}$ receptor, in which the former is reported to be an antagonist, whereas the latter is an indirect agonist (with an unreported affinity) (Russo et al., 2005; Cascio et al., 2010; Rock et al., 2011, 2012). There are currently no data in this regard for $\triangle 9$-THC.

Table 2 provides a more detailed analysis of the physiologic activities that are exhibited by CBG (whether in intact animals, whole cells, or subcellular preparations). CBG appears to act as an agonist at the $\alpha-2$ receptor (with varying $\mathrm{EC}_{50}$ values reported) and all of the Transient Receptor Potential Cation Channel (TRP) family channels (except TRPM8, at which it is reported to act as an antagonist). Together, these data suggest that the physiologic effects of CBG tend toward Gi-mediated inhibition, autoregulatory activity, and calcium-based signaling by both ion channels and protein kinase $\mathrm{C}(\mathrm{PKC})$. Given the differential pharmacological profiles, there are a number of important concerns to consider concerning the therapeutic potentials and potential adverse outcomes for the widespread and unregulated use of CBG.

\section{The $\alpha$-2 Adrenergic Receptor (the $\alpha-2$ Adrenoceptor)}

The observation that CBG is a potent agonist at the $\alpha-2$ receptor has significant implications for potential therapeutic uses and adverse side effects (Cascio et al., 2010). Catecholamines (dopamine, norepinephrine, and epinephrine), produced by the nervous system, use a variety of methods to exert different physiologic effects. The main receptor families that mediate these functions are the adrenergic receptors [alpha $(\alpha-1$ and $\alpha-2)$ and beta $(\beta-1, \beta-2$, and $\beta-3)$ families] and the dopamine receptors (D1 through D5 receptors) (Molinoff, 1984). Using the norepinephrine system as an exemplar, these receptors almost all act in the following way: vesicles containing norepinephrine are released from the presynaptic neuron, and synaptic norepinephrine activates postsynaptic G protein-coupled receptors (GPCRs) to create a unique downstream effect. Although the catecholamine receptor families are all GPCRs of varying functions, the $\alpha-2$ receptor class is best known as a presynaptic receptor (Saunders and Limbird, 1999); however, it also functions in peripheral tissues and cells. Extensive study of this autoregulatory receptor has shown its ability to dampen sympathetic nervous system activity through its Gi (inhibitory) activity that is triggered when synaptic norepinephrine binds to the presynaptic receptor. Additionally, $\alpha-2$ receptor activation demonstrates

TABLE 1

Pharmacodynamic properties of $\triangle 9$-THC, CBD, and CBG at cannabinoid receptors

Binding affinities for $\triangle 9$-THC, CBD, and CBG at the two canonical cannabinoid receptors (CB1 and CB2), as well as a third receptor, GPR55 (commonly referred to as CB3). $\triangle 9$-THC acts as an agonist at all three receptors, whereas CBD acts as an antagonist; CBG acts as a weak or partial agonist at CB1 and CB2, and its function at GPR55 is currently unknown.

\begin{tabular}{|c|c|c|c|c|c|c|}
\hline \multirow[b]{2}{*}{ Receptor } & \multicolumn{2}{|c|}{$\Delta 9$-THC } & \multicolumn{2}{|r|}{$\mathrm{CBD}$} & \multicolumn{2}{|l|}{ CBG } \\
\hline & Affinity & Function & Affinity & Function & Affinity & Function \\
\hline & $n M$ & & $n M$ & & $n M$ & \\
\hline CB1 & $5.1-80.3(\mathrm{Ki}) a, b$ & Partial agonist & $1458.5-4900(\mathrm{Ki}) a, b$ & Inverse agonist/antagonist & $440-1045(\mathrm{Ki})^{b}, c, d, e$ & Weak agonist \\
\hline CB2 & $3.1-75.3(\mathrm{Ki}) a, b$ & Agonist & $372.4-4200(\mathrm{Ki}) a, b$ & Inverse agonist & $153.4-1225(\mathrm{Ki}) b, c, d, e$ & Partial agonist \\
\hline GPR55 & $8\left(\mathrm{EC}_{50}\right) f$ & Agonist & $445\left(\mathrm{IC}_{50}\right) f$ & Antagonist & N.T. & Unknown \\
\hline
\end{tabular}

N.T., not tested.

${ }^{a}$ Pertwee (2008)

${ }^{b}$ Rosenthaler et al. (2014).

${ }^{c}$ Cascio et al. (2010).

${ }^{d}$ Pollastro et al. (2011).

${ }^{e}$ Navarro et al. (2018).

${ }_{\text {Ryberg et al. (2007). }}$ 
TABLE 2

Pharmacodynamic properties of $\triangle 9-\mathrm{THC}, \mathrm{CBD}$, and CBG at noncannabinoid receptors

Binding affinities for $\triangle 9$-THC, CBD, and CBG at TRP ion channels, $\alpha-2$ adrenoceptors, the serotonin receptor 5-HT $1 \mathrm{~A}$, and PPAR $\gamma$ are presented. Values are all $\mathrm{EC}_{50}$ for agonists and $\mathrm{IC}_{50}$ for antagonists.

\begin{tabular}{|c|c|c|c|c|c|c|}
\hline \multirow[b]{2}{*}{ Receptor } & \multicolumn{2}{|c|}{$\Delta 9$-THC } & \multicolumn{2}{|c|}{ CBD } & \multicolumn{2}{|c|}{ CBG } \\
\hline & Affinity & Function & Affinity & Function & Affinity & Function \\
\hline & $n M$ & & $n M$ & & $n M$ & \\
\hline TRPA1 & $230 a, b$ & Agonist & $110 a, b$ & Agonist & $700 a, b, c$ & Agonist \\
\hline TRPV1 & N.D. $a, b$ & Unknown & $1000 a, b$ & Agonist & $1300 a, b, c$ & Agonist \\
\hline TRPV2 & $650 a, b$ & Agonist & $1250 a, b$ & Agonist & $1720 a, b, c$ & Agonist \\
\hline TRPV3 & $9500 b, d$ & Agonist & $3700 b, d$ & Agonist & $1000 b, d$ & Agonist \\
\hline TRPV4 & $850 b, d$ & Agonist & $800 b, d$ & Agonist & $5100 b, d$ & Agonist \\
\hline TRPM8 & $160 a, b$ & Antagonist & $140 a, b$ & Antagonist & $160 a, b, c$ & Antagonist \\
\hline a-2 adrenoceptor & N.T. & Unknown & N.T. & Unknown & $0.2-72.8 e$ & Agonist \\
\hline $5-\mathrm{HT}_{1 \mathrm{~A}}$ & N.T. & Unknown & N.D.f.g & Indirect agonist & $51.9 d, e$ & Antagonist \\
\hline $\operatorname{PPAR} \gamma$ & $2120 h$ & Agonist & $2010 h$ & Agonist & $1270 h$ & Agonist \\
\hline
\end{tabular}

N.D., not detected; N.T., not tested.

${ }^{a}$ De Petrocellis et al., 2011.

${ }^{b}$ Muller et al., 2019.

${ }^{c}$ Pollastro et al., 2011.

${ }^{d}$ Rock et al., 2011.

${ }^{e}$ Cascio et al., 2010.

$f_{\text {Russo et al., } 2005 .}$

${ }^{g}$ Rock et al., 2012.

${ }^{h}$ Granja et al., 2012.

other mechanisms of reduced sympathetic activity such as opening receptor operated $\mathrm{K}+$ channels and inhibiting voltagegated calcium channels (Cotecchia et al., 1990; Saunders and Limbird, 1999). The family of $\alpha-2$ receptors has its own further classification into three receptor subtypes- $\alpha-2 \mathrm{~A}, \alpha-2 \mathrm{~B}$, and $\alpha-2 \mathrm{C}$ - the genes for which are found on chromosomes 10, 2, and 4, respectively (Saunders and Limbird, 1999).

Although highly related, the different subtypes of the $\alpha$-2 receptor are distinct in their sequence, structure, and receptor distribution in the body (Saunders and Limbird, 1999). Structural differences between subtypes arise from the intraand extracellular regions of the receptor structure, as well as differential post-translational modifications. Anatomic differences are well characterized for the three subtypes in the brain and in the periphery through in situ hybridization of receptor mRNA and through immunochemical analysis of rodent receptor locations (Saunders and Limbird, 1999). In the brain, $2 \mathrm{~A}$ receptors are widely distributed and abundant in the locus coeruleus and in brainstem regions with homeostatic functions. The $\alpha$-2B receptors are found in the thalamic nuclei, whereas $2 \mathrm{C}$ is found in the basal ganglia, olfactory tubercle, hippocampus, and cerebral cortex (Molinoff, 1984; Arnsten et al., 1988; Saunders and Limbird, 1999). The 2A and 2C receptors are the primary contributors of $\alpha$-2 receptor function in the central nervous system. Although all three subtypes are found in the nervous system, each subtype has a unique peripheral tissue distribution. In the periphery, $2 \mathrm{~A}$ is found in platelets, beta cells of the pancreas, adrenal glands, intestinal epithelia, vascular endothelium, and smooth muscle cells (Molinoff, 1984; Saunders and Limbird, 1999); $\alpha-2 \mathrm{~B}$ is found in rat neonatal lung and liver, the adult kidney, vascular endothelium, and smooth muscle cells, whereas $2 \mathrm{C}$ is only found in the adult kidney (Molinoff, 1984; Saunders and Limbird, 1999).

Many of the discoveries involving $\alpha$-2 receptor function were derived from experimental administration of known $\alpha-2$ agonists, such as clonidine, and measuring local and systemic effects. Current therapeutic interest in peripheral $\alpha$-2 agonism effects center around their antihypertensive, sedative, and analgesic functions. Clonidine and dexmedetomidine (a newer and more selective $\alpha$-2 full agonist) are often used in anesthesia settings (Hunter et al., 1997; Gertler et al., 2001). Clonidine was originally popular as an antihypertensive agent but is now considered second-line or third-line in an antihypertensive regimen because of its effects at other nonadrenergic receptors. The vasodilatory and hypotensive effects of clonidine are multifactorial, owing to clonidine's actions at not only the $\alpha-2$ receptors but also imidazoline receptors, both of which reduce vascular tone when activated (Ernsberger et al., 1990). Another $\alpha-2$ agonist, guanfacine, has higher selectivity at the $\alpha-2 \mathrm{~A}$ receptor and less activity at imidazoline receptors. When compared with clonidine administration, guanfacine administration reduces blood pressure less than clonidine (Arnsten et al., 1988). Although $\alpha-2$ agonists are not indicated in most antihypertensive regimens today because of efficacy of other drugs, clonidine and guanfacine are commonly prescribed for neuropsychiatric diseases because of their effects on $\alpha-2$ receptors in the prefrontal cortex. The role of $\alpha-2$ agonists in neuropsychiatric disease depends on their ability to modulate and improve impaired prefrontal cortex (PFC) functioning (Arnsten et al., 1988; Arnsten, 2010). PFC impairment is a common finding during normal aging, as well as conditions like attention-deficit hyperactivity disorder (ADHD), tic disorders, post-traumatic stress disorder, dementia, and others (Arnsten, 2010). The $\alpha-2$ receptors, specifically $2 \mathrm{~A}$, are heavily involved in norepinephrine signaling in the PFC, and $\alpha-2$ agonists are used to improve working memory and planning ability in ADHD in children and adults, along with tic disorders and reduction of opiate withdrawal symptom severity (Arnsten, 2010). In the treatment of ADHD, $\alpha-2$ agonists have the additional benefit of being effective alternatives or adjuncts to the first-line treatment: stimulant medications. Use of $\alpha-2$ agonists in conjunction with stimulant medications can reduce stimulant-induced tics and hypertension, along with reducing the necessary dosing to achieve symptom management for stimulant-sensitive patients (Arnsten et al., 1988; Arnsten, 2010). Clonidine and guanfacine, both $\alpha-2$ agonists, have gained popularity in treatment of 
psychiatric disorders and are another example of $\alpha$-2 subtype specificity determining functionality; guanfacine has less intense hypotensive and sedative side effects than clonidine while boasting increased activity at the PFC (Arnsten, 2010). Researchers believe that this is due to its specificity at $\alpha-2 \mathrm{~A}$. Interestingly, whereas agonism of $\alpha$-2A receptors has shown benefits in selected psychiatric disorders, antagonism of $\alpha$-2C receptors may be beneficial in other psychiatric disorders, such as psychosis and schizophrenia (Uys et al., 2017). This diversity in receptor distribution and potential function indicates a major need for further research into subtypespecific actions and functions of the $\alpha-2$ adrenergic receptors.

Although $\alpha-2$ agonists have considerable therapeutic applications, the current knowledge of CBG activity at $\alpha-2$ receptor subtype and location is lacking. From the potency of CBG at this adrenergic receptor, ingestion may unpredictably change blood pressure, induce sedation, and interact with other cardiovascular medications. Later in this review, we discuss the imperatives for further research into these physiologic effects of CBG administration.

\section{The Serotonin 5- $\mathrm{HT}_{1 \mathrm{~A}}$ Receptor}

Serotonin, or 5-hydroxytryptamine (5-HT), is a monoamine neurotransmitter produced throughout the body for a variety of physiologic and neurologic functions. It plays a central role in maintaining homeostatic functions (e.g., in the enteric gastrointestinal nervous system) (Coates et al., 2017). In the central nervous system, it is a target of antidepression medications. Serotonin binds to different receptor families (Nichols and Nichols, 2008); here, we will focus on the 5-HT $1 \mathrm{~A}$ receptor, which has previously been shown to interact with endogenous and exogenous cannabinoid ligands. The $5-\mathrm{HT}_{1 \mathrm{~A}}$ receptor is a Gi/o GPCR that inhibits adenylate cyclase, but the receptor has also been shown to interact with other growth factor pathways (Rojas and Fiedler, 2016). The receptor is located both pre- and postsynaptically, and the downstream functions of receptor activation vary based on neuronal identity and location. Inhibition of $5-\mathrm{HT}_{1 \mathrm{~A}}$ autoreceptor (presynaptic) activity is suggested to significantly affect the speed and efficacy of other serotonin-modulating drugs, such as selective serotonin reuptake inhibitors (Artigas et al., 1996). A selective $5-\mathrm{HT}_{1 \mathrm{~A}}$ antagonist, WAY100635, has potentiated the effects of fluoxetine, a selective serotonin reuptake inhibitor, in vivo. Additionally, the $\beta$-adrenergic/5- $\mathrm{HT}_{1 \mathrm{~A}}$ antagonist pindolol was found to enhance the effects of several different classes of serotoninergic antidepressants in patients with major depressive disorder (Artigas et al., 1994). One possible mechanism for these effects is that $5-\mathrm{HT}_{1 \mathrm{~A}}$ antagonists reduce the increase in $5-\mathrm{HT}_{1 \mathrm{~A}}$ autoreceptor activity created by antidepressants, which increases synaptic serotonin availability.

In the present context, we note that $\mathrm{CBG}$ has been reported to be a potent $(50 \mathrm{nM}) 5-\mathrm{HT}_{1 \mathrm{~A}}$ antagonist. Given this pharmacological characteristic, it may have unpredictable potentiating effects on concurrently administered psychiatric medications and serotonin-modulating substances. Recently, Echeverry et al. (2020) reported that CBG and CBD have neuroprotective effects against oxidative neurotoxicity through a $5-\mathrm{HT}_{1 \mathrm{~A}}$ receptor-mediated mechanism. The effects of CBG on serotonin signaling or availability have not yet been thoroughly studied, and more research is needed to understand the impact of CBG-mediated $5-\mathrm{HT}_{1 \mathrm{~A}}$ antagonism before it is made widely available in an unregulated commercial environment.

\section{The Peroxisome Proliferator-Activated Receptors}

The PPAR family is a collection of nuclear receptor transcription factors; there are three isoforms: $\operatorname{PPAR} \alpha, \operatorname{PPAR} \beta$, and PPAR $\gamma$. PPAR nuclear activation induces conformational changes and binding to PPAR response elements of DNA to modulate gene transcription (O'Sullivan and Kendall, 2010; O'Sullivan, 2016). These receptors transcriptionally regulate lipid metabolism, hepatic metabolic functions, and inflammation. PPARs accept a wide variety of ligands because of their large binding domains, and many cannabinoids and their metabolites are reported to interact with the various isoforms (O'Sullivan and Kendall, 2010; O'Sullivan, 2016). In the reviewed studies, CBG exhibits stronger affinity to the PPAR $\gamma$ receptor than $\triangle 9$-THC and CBD.

$\operatorname{PPAR} \gamma$ will regulate adipocyte differentiation, insulin sensitivity, and inflammatory states. This is the therapeutic mechanism of thiazolidinediones, like rosiglitazone, commonly used in patients with type 2 diabetes to improve adipocyte functioning and increase insulin sensitivity (O'Sullivan and Kendall, 2010; O'Sullivan, 2016). Cannabinoid compounds display varying affinities at PPAR isoforms, and the effects of CBG on these receptors are being studied in the regulation of inflammation and metabolic functioning; these potential therapeutic effects are reviewed in the next section.

\section{Potential Therapeutic Potentials for CBG}

Based on the receptor signaling of the $\alpha-2,5-\mathrm{HT}_{1 \mathrm{~A}}$, and PPAR $\gamma$ receptors and the reported affinities of CBG at these receptors (in the tens of nanomolars to sub-nanomolar range), there are many reasons to believe that CBG will have therapeutic potential (Cascio et al., 2010; Rock et al., 2011; Granja et al., 2012). Similarly, however, there are reasons to monitor high-dose CBG for untoward side effects beyond drugdrug interactions.

Neuroprotection and Neuromodulation. A number of studies have shown that CBG and a second-generation synthetic quinone derivative, VCE-003.2, have neuroprotective potential in vitro and in animal models to reduce the severity of neurologic illnesses, such as Huntington disease (HD), amyotrophic lateral sclerosis, Parkinson disease, and multiple sclerosis; this seems to largely be mediated through PPAR $\gamma$ (Granja et al., 2012; Carrillo-Salinas et al., 2014; DíazAlonso et al., 2016; Mammana et al., 2019). VCE-003.2 is distinct from the first-generation CBG quinone derivative VCE-003 because it retains neuroprotective activity through dose-dependent PPAR $\gamma$ activation but does so without the cytotoxicity and thiophilic properties of VCE-003 (Díaz-Alonso et al., 2016). These properties lend some pharmacological explanation to its beneficial effects on various models of neurotoxicity. Indeed, VCE-003.2 is reported to have negligible affinity at either CB1 or CB2 $(>40 \mu \mathrm{M})$. CBG and VCE003.2 have been shown to reduce the inflammatory molecules TNF- $\alpha$, IL- $1 \beta$, IL-6, Macrophage Inflammatory Protein (MIP$1 \alpha$ ), and Prostaglandin E2 (PGE2) in rat microglial cells treated with lipopolysaccharide (LPS), and both compounds reduce glutamate-induced oxidative cell death in mouse hippocampal cells (Granja et al., 2012). 
Using two mouse models of HD, Valdeolivas et al. (2015) reported improvement of HD symptoms. In particular, in 3nitropropionate-treated mice, CBG prevented striatal neuron death, reduced markers of inflammation, and improved motor deficits (Valdeolivas et al., 2015). The results in a transgenic R6/2 mouse model of HD were not as robust. Treatment with CBG did not reduce the weight loss observed in this model, but it did moderately improve Rotarod performance and reduced the number of huntingtin aggregates (Valdeolivas et al., 2015). A study using VCE-003.2 found a similar improvement in HD symptoms in the murine model of HD (Díaz-Alonso et al., 2016). In the quinolinic acid murine model of HD, treatment with VCE-003.2 improved Rotarod performance (Díaz-Alonso et al., 2016). VCE-003.2 also improved motor deficits in the 3-nitropropionate (NP3) model of HD, and this was found to be dependent upon PPAR $\gamma$ activity in that inhibition of PPAR $\gamma$ by T0070907 blocked the effect of VCE-003.2 treatment (Díaz-Alonso et al., 2016).

Experiments using the SOD ${ }^{\mathrm{G} 93 \mathrm{~A}}$ mouse model of amyotrophic lateral sclerosis found that VCE-003.2 improved the neuropathological symptoms, including attenuating weight loss, improving clinical scores, and preserving motor neurons in the spinal cord (Rodríguez-Cueto et al., 2018). Additionally, astrocytes cultured from $\mathrm{SOD}^{\mathrm{G} 93 \mathrm{~A}}$, when treated with VCE003.2, exhibited a morphology close to wild-type astrocytes (Rodríguez-Cueto et al., 2018). Using an lipopolysaccharide (LPS)-induced inflammatory model of Parkinson disease, VCE003.2 reduced the inflammatory markers $\mathrm{TNF}-\alpha, \mathrm{IL}-1 \beta$, and inducible nitric oxide synthase (iNOS), and this response was mediated through PPAR $\gamma$ (García et al., 2018). Finally, VCE003.2 has also been shown to improve motor activity, reduce vascular cell adhesion molecule (VCAM-1) expression, and decrease microglia activity in the Theiler murine encephalomyelitis virus model of multiple sclerosis (Granja et al., 2012).

Another area of interest is the therapeutic potential of CBG involving $5-\mathrm{HT}_{1 \mathrm{~A}}$ receptor activity. In 2020, Echeverry et al. (2020) studied the effects of CBG and CBD on neurotoxicity as assessed in neural cell cultures. The neuroprotective qualities of CBD and CBG were distinct at two different models of oxidative damage (the $\mathrm{H}_{2} \mathrm{O}_{2}$ model and the rotenone model) (Echeverry et al., 2020). The authors found that the protective effects were lost when CBG and CBD were administered with a $5-\mathrm{HT}_{1 \mathrm{~A}}$ antagonist, WAY-100635, but were unchanged with CB1 and CB2 receptor antagonists (Echeverry et al., 2020). This suggests that the protective effects of CBG and CBD against oxidative neurotoxicity are derived from a $5-\mathrm{HT}_{1 \mathrm{~A}}$ receptor-mediated process (Echeverry et al., 2020).

Finally, a relatively unexplored area of CBG neuromodulation is its effect on neurotransmission. Recently, transcriptomic changes by administration of CBD and CBG on motor neuron-like cells has shown an insight into this neuromodulatory effect. In NSC-34 motor neuron-like cells, authors Gugliandolo et al. (2020) compared the transcriptomic changes from CBD and CBG. The two compounds similarly changed transcription in the dopamine, GABA, and glutamate pathways. This was from, generally, reduced expression of glutamate release genes, enhanced expression of GABA release genes, and upregulated dopamine D4 receptor and its downstream effectors (Gugliandolo et al., 2020). Characterization of these changes on behavior and neuronal signaling through translational research is necessary to better understand the context of CBG in psychopharmacology.
However, the results do speak to the need to monitor untoward side effects.

Gastrointestinal Disease. Cannabigerol has been explored as a therapeutic for gastrointestinal diseases such as colorectal cancer and colitis using mouse models. In the dinitrobenzene sulfuric acid model of colitis, treatment with CBG increased the rate of tissue recovery in the colon as measured by histologic structure, the ratio of colon weight to length, colonic permeability, and reduced inflammation (Borrelli et al., 2013). The authors also found that CBG was also effective as a treatment to prevent colitis-associated damage (Borrelli et al., 2013). In a follow-up study, it was found that cannabigerol reduced tumor formation in the azoxymethane model of colorectal cancer and reduced xenograft tumor growth (Borrelli et al., 2014). Although the authors conclude that this reduction was mediated by TRPM8, these studies were performed in cell culture rather than in the murine model. In 2020, Pagano et al. (2020) used a mouse model of dinitrobenzene sulfuric acid-induced colitis to study the effects of CBG on inflammatory activity. Orally administered CBG reduced colonic inflammation as measured by significantly reducing myeloperoxidase (MPO) activity, IL-1b levels, and serum fluorescein isothiocyanate (FITC)-dextran concentration. In contrast, CBD on its own did not induce significant changes to these metrics. Adding fish oil to the treatment paradigm increased the CBG-induced reduction in myeloperoxidase activity, IL-1b levels, and serum FITC-dextran concentration (Pagano et al., 2020).

CBG has also been shown to increase feeding in rats and to reduce weight loss associated with cisplatin chemotherapy (Brierley et al., 2016, 2017, 2019). However, an earlier study found no impact of CBG on feeding behavior (Farrimond et al., 2012). Unlike $\Delta 9$-THC and CBD, CBG has not been found to have antiemetic effects and appears to oppose the antiemetic effects of CBD (Rock et al., 2011). Taken together, these studies indicate that there may be a role for cannabigerol in chemotherapy-associated weight loss and loss of appetite, although $\Delta 9$-THC (dronabinol) and nabilone have already been proven efficacious, CBG lacks the potential euphoric side effect of these medications.

Metabolic Syndrome. Metabolic syndrome affects millions of Americans and contributes to the highest burden of healthcare costs and preventable mortality in the country. A clinical diagnosis, metabolic syndrome is a combination of insulin resistance, obesity, hypertension, high levels of lowdensity lipoprotein, and reduced levels of high-density lipoprotein. Although a wide array of medications and surgical interventions are available for metabolic syndrome, few treatments are effective enough to serve as monotherapy, and many patients need multiple medications with harsh side effects to keep this chronic illness at bay.

Recent studies on CBG provide promise for its use as part of a multifactorial pharmacotherapy for metabolic syndrome and its components. Hypertension, one component of metabolic syndrome, can be modestly reduced with $\alpha$-2 agonist therapy, which reduces synaptic norepinephrine levels to reduce vasoconstriction and improve blood pressure. CBG is currently the only known cannabinoid that is an agonist at the adrenergic receptor (Cascio et al., 2010). In addition, as previously mentioned, CBG and its derivatives are shown to act on PPAR $\gamma$ receptors in its role on neuroinflammation. In 
2019, in vitro modeling of phytocannabinoids suggested CBG as a dual PPAR $\alpha / \gamma$ agonist, first through computer modeling and prediction and then confirmation in HepG2 (human liver epithelial-like) and 3T3L1 (mouse embryo fibroblast) cell lines (D’Aniello et al., 2019).

More support for PPAR $\alpha / \gamma$ agonism of CBG came in 2020 from researchers who tested PPAR $\alpha / \gamma$-dependent differentiation in bone marrow mesenchymal stem cell lines (Fellous et al., 2020). This effect was enhanced when combined with $\mathrm{CBD}$ and had a similar effect as a commonly prescribed drug for metabolic syndrome, rosiglitazone (Fellous et al., 2020). Rosiglitazone is known to act as an agonist at PPAR $\gamma$ receptors and to increase expression of glucose transporter type 4 (GLUT4) glucose channels; both of these effects increase insulin sensitivity. $\mathrm{CBG}$ and the $\mathrm{CBG} / \mathrm{CBD}$ combinations provided similar effects in vivo as rosiglitazone and additive effects to improve adipogenesis (Fellous et al., 2020). Renewed adipogenesis and insulin sensitization improve both symptoms and pathology of metabolic syndrome; therefore, CBG could be explored as a potential therapeutic for this devastating disease.

Antibacterial Agent. A number of cannabinoids have been reported to have antibacterial activity; however, CBG was found to be among the most potent cannabinoids tested against antibiotic resistant strains of Staphylococcus aureus (Appendino et al., 2008). In comparison with conventional antibiotics, CBG had a lower minimum inhibitory concentration than norfloxacin in five of the six strains tested and was more potent than erythromycin, tetracycline, and oxacillin in at least one resistant strain (Appendino et al., 2008). Using a systemic $S$. aureus infection model in mice, Farha et al. (2020) showed that CBG was as effective at reducing colony forming units as vancomycin. This study also found that CBG was effective against Gram negative bacteria only when the outer membrane was first permeabilized, suggesting that the outer membrane of Gram negative bacteria presents a permeability problem for cannabinoids (Farha et al., 2020). Using in silico modeling, Pinzi et al. (2019) found that CBG acts as an inhibitor of enoyl acyl carrier protein reductase (Gene Name; InhA), and they verified their model with in vitro testing and found that $\mathrm{CBG}$ inhibits enoyl acyl carrier, with an $\mathrm{IC}_{50}$ value in the low micromolar range. These data are encouraging because there is a need to develop novel therapeutics, as antibiotic resistance in bacteria is a continuing healthcare issue.

\section{Potential Arguments against CBG as a Therapeutic}

We have highlighted the potential of cannabigerol as a therapeutic and in medical research; however, for it to be seriously considered as a potential therapeutic, it must be rigorously tested for safety and unintended effects.

CBG has potent activity at the $\alpha$-2 receptor, and this unique property could also induce unintended cardiovascular consequences such as hypotension, bradycardia, and xerostomia. Additionally, some investigators have reported hypertension as a counterintuitive adverse effect in high doses of $\alpha-2$ agonists, which appears to be mediated by the $\alpha-2 \mathrm{~B}$ receptor subtype (Philipp et al., 2002). The potential for this adverse effect is unclear in the case of CBG, since its activity at different $\alpha-2$ receptor subtypes has yet to be studied. Although we surmise that CBG may have therapeutic potential among neurologic, gastrointestinal, and metabolic disorders, there must be more research to ensure that unintended cardiovascular effects do not reduce the utility of CBG. In addition, in this era of unregulated CBD preparations, companies are making unsubstantiated claims and overselling the benefits and underselling the risks. Indeed, companies are already touting CBG as the "mother of all cannabinoids," presumably because it is the immediate precursor of CBD and $\Delta 9$-THC. What they fail to point out, however, is that only the Cannabis plant goes on to convert CBG to the other molecules - the human body does not. This is problematic because very few Cannabis strains actually harbor large concentrations of CBG, so there is not much prior reporting (and virtually no documentation) of human side effects of CBG.

Several recent cautionary situations come to mind. In a couple of recent reports on CBD oil, it has been reported that not all CBD oil preparations had concentrations of CBD close to what the manufacturer claims (Bonn-Miller et al., 2017; Pavlovic et al., 2018; Raup-Konsavage et al., 2020; Urasaki et al., 2020). Moreover, the U.S. Food and Drug Administration had to prompt recalls for CBD preparations that contained unacceptable concentrations of lead (https:// www.fda.gov/safety/recalls-market-withdrawals-safety-alerts/ inhe-manufacturing-llc-and-mhr-brands-issues-voluntarynationwide-recall-several-products-due?utm_campaign=FDA $\% 20$ MedWatch\%20-\%20Several\%20Hemp\%20Oil\%20Products $\% 20$ from $\% 20 \mathrm{InHe} \% 20$ Manufacturing $\% 20$ and $\% 20 \mathrm{MHR} \%$ 20Brands\%3A\%20Recall\&utm_medium =email\&utm_source= Eloqua; https://www.fda.gov/safety/recalls-market-withdrawalssafety-alerts/summitt-labs-issues-voluntary-nationwide-recallkore-organic-watermelon-cbd-oil-due-high-lead). We have reported that, even when the different CBD oil preparations contain the reported levels of this phytocannabinoid, they had very different activities in suppressing cancer cell growth in vitro, and only one approximated the activity of pure CBD (Raup-Konsavage et al., 2020). Finally, there is also the very real risk of inducing drug-drug interactions when over-the-counter preparations like CBD oil or CBG oil are taken by a patient on other (prescription) medications (Kocis and Vrana, 2020). This may lead to unintended adverse consequences when not appropriately monitored.

\section{Future Imperatives}

Research into cannabigerol is in its infancy but has shown promise for addressing a diverse array of therapeutic needs. Based on its pharmacodynamics, here we highlight potential indications for CBG and its derivatives to improve available drug treatment regimens for selected diseases and medical conditions. However, these applications will rely on additional research studies to further understand how CBG can be used safely and effectively.

First, there is potential for CBG as a major player in the treatment of metabolic disease as described by its action on the PPAR family of receptors to improve insulin sensitivity and adipogenesis. A supplementary effect is its antihypertensive properties at $\alpha-2$ receptors. Patients with diabetes frequently have hypertension, hyperlipidemia, and insulin resistance stemming from glucose dysregulation and vascular endothelial dysfunction. CBG may improve this profile as an adjunct to the mainstay of treatment, metformin, or potentially serve as its own regimen. In current practice, $\alpha-2$ agonists such as clonidine are infrequently used as antihypertensive agents, largely because these effects of clonidine 
are enhanced with imidazoline receptor activity; this rendered clonidine too powerful as an antihypertensive drug and unreliable in practice. To our knowledge, the sympatholytic effect of CBG is limited to $\alpha$ - 2 activity, which makes it more useful for this indication than clonidine, but more studies are needed.

Second, several studies have described the neuroprotective effects of CBG through action on the PPAR family of receptors. Other sources have reported reduction in age-related cognitive decline in patients with neurodegenerative disease with the addition of $\alpha-2$ agonists to their treatment regimens. Although CBG's effect on cognition has yet to be studied, it may play a role for improving quality of life in these vulnerable populations, as the few drugs currently available for neurodegenerative diseases also carry uncomfortable and disabling side effects.

Third, similar to other phytocannabinoid derivatives, CBG may play an important role for improving the drug cocktails of patients who struggle with disorders of executive function, such as schizophrenia and ADHD. Two current $\alpha-2$ agonists, clonidine and guanfacine, are indicated for their $\alpha-2-$ mediated action on the human prefrontal cortex to improve executive function and self-regulation; however, clonidine is less safely prescribed because of its potent antihypertensive properties and generalized action on $\alpha-2$ receptor subtypes. Guanfacine is well studied as an adjunct therapy with stimulants in ADHD because of its $\alpha-2 \mathrm{~A}$ receptor subtype specificity. The subtype specificity of CBG has yet to be elucidated; therefore, it cannot be predicted how CBG will improve executive dysfunction compared with guanfacine. Finally, researchers have studied the effects of CBG as a safe appetite stimulant in chemotherapy-related appetite suppression in vivo and as an agent that reduces in vitro signs of pathology in colitis and colorectal cancer.

In closing, although there is much to suggest that CBG may provide alternative therapeutics for a number of disorders, much is left to learn. In particular, given the potent bioactivity CBG displays in a number of settings, we should be very cautious about releasing it in an unregulated retail environment. There is simply insufficient experience with this relatively rare phytocannabinoid, and the potential for adverse effects is high. Given the dramatic increase in unregulated $\mathrm{CBD}$ oil use following deregulation of hemp, it behooves the pharmacology community to undertake CBG research before its use explodes as well.

\section{Acknowledgments}

The authors thank Dr. Dhimant Desai for chemical insights into CBG synthesis and Bradley Winters for preparation of Fig. 1.

\section{Authorship Contributions}

Wrote or contributed to the writing of the manuscript: Nachnani, Raup-Konsavage, Vrana.

\section{References}

Ambrose T and Simmons A (2019) Cannabis, cannabinoids, and the endocannabinoid system-is there therapeutic potential for inflammatory bowel disease? $J$ Crohns Colitis 13:525-535.

Appendino G, Gibbons S, Giana A, Pagani A, Grassi G, Stavri M, Smith E, and Rahman MM (2008) Antibacterial cannabinoids from Cannabis sativa: a structure-activity study. $J$ Nat Prod 71:1427-1430.

Arnsten AF (2010) The use of $\alpha-2 \mathrm{~A}$ adrenergic agonists for the treatment of attentiondeficit/hyperactivity disorder. Expert Rev Neurother 10:1595-1605.

Arnsten AF, Cai JX, and Goldman-Rakic PS (1988) The alpha-2 adrenergic agonist guanfacine improves memory in aged monkeys without sedative or hypotensive side effects: evidence for alpha-2 receptor subtypes. J Neurosci 8:4287-4298.
Artigas F, Perez V, and Alvarez E (1994) Pindolol induces a rapid improvement of depressed patients treated with serotonin reuptake inhibitors. Arch Gen Psychiatry 51:248-251.

Artigas F, Romero L, de Montigny C, and Blier P (1996) Acceleration of the effect of selected antidepressant drugs in major depression by 5 -HT1A antagonists. Trends Neurosci 19:378-383.

Baron EP (2018) Medicinal properties of cannabinoids, terpenes, and flavonoids in cannabis, and benefits in migraine, headache, and pain: an update on current evidence and cannabis science. Headache 58:1139-1186.

Bonn-Miller MO, Loflin MJE, Thomas BF, Marcu JP, Hyke T, and Vandrey R (2017) Labeling accuracy of cannabidiol extracts sold online. JAMA 318: 1708-1709.

Borrelli F, Fasolino I, Romano B, Capasso R, Maiello F, Coppola D, Orlando P, Battista G, Pagano E, Di Marzo V, et al. (2013) Beneficial effect of the nonpsychotropic plant cannabinoid cannabigerol on experimental inflammatory bowel disease. Biochem Pharmacol 85:1306-1316.

Borrelli F, Pagano E, Romano B, Panzera S, Maiello F, Coppola D, De Petrocellis L, Buono L, Orlando P, and Izzo AA (2014) Colon carcinogenesis is inhibited by the TRPM8 antagonist cannabigerol, a Cannabis-derived non-psychotropic cannabinoid. Carcinogenesis 35:2787-2797.

Bostwick JM (2012) Blurred boundaries: the therapeutics and politics of medical marijuana. Mayo Clin Proc 87:172-186.

Brierley DI, Harman JR, Giallourou N, Leishman E, Roashan AE, Mellows BAD, Bradshaw HB, Swann JR, Patel K, Whalley BJ, et al. (2019) Chemotherapyinduced cachexia dysregulates hypothalamic and systemic lipoamines and is attenuated by cannabigerol. J Cachexia Sarcopenia Muscle 10:844-859.

Brierley DI, Samuels J, Duncan M, Whalley BJ, and Williams CM (2016) Cannabigerol is a novel, well-tolerated appetite stimulant in pre-satiated rats. Psychopharmacology (Berl) 233:3603-3613.

Brierley DI, Samuels J, Duncan M, Whalley BJ, and Williams CM (2017) A cannabigerolrich Cannabis sativa extract, devoid of [INCREMENT]9-tetrahydrocannabinol, elicits hyperphagia in rats. Behav Pharmacol 28:280-284.

Carrier EJ, Auchampach JA, and Hillard CJ (2006) Inhibition of an equilibrative nucleoside transporter by cannabidiol: a mechanism of cannabinoid immunosuppression. Proc Natl Acad Sci USA 103:7895-7900.

Carrillo-Salinas FJ, Navarrete C, Mecha M, Feliú A, Collado JA, Cantarero I, Bellido ML, Muñoz E, and Guaza C (2014) A cannabigerol derivative suppresses immune responses and protects mice from experimental autoimmune encephalomyelitis. PLoS One 9:e94733.

Cascio MG, Gauson LA, Stevenson LA, Ross RA, and Pertwee RG (2010) Evidence that the plant cannabinoid cannabigerol is a highly potent alpha2-adrenoceptor agonist and moderately potent 5HT1A receptor antagonist. $\mathrm{Br} J$ Pharmacol 159: $129-141$.

Coates MD, Tekin I, Vrana KE, and Mawe GM (2017) Review article: the many potential roles of intestinal serotonin (5-hydroxytryptamine, 5-HT) signalling in inflammatory bowel disease. Aliment Pharmacol Ther 46:569-580.

Cotecchia S, Kobilka BK, Daniel KW, Nolan RD, Lapetina EY, Caron MG, Lefkowitz RJ, and Regan JW (1990) Multiple second messenger pathways of alphaadrenergic receptor subtypes expressed in eukaryotic cells. J Biol Chem 265: 63-69.

D'Aniello E, Fellous T, Iannotti FA, Gentile A, Allarà M, Balestrieri F, Gray R, Amodeo P, Vitale RM, and Di Marzo V (2019) Identification and characterization of phytocannabinoids as novel dual PPAR $\alpha / \gamma$ agonists by a computational and in vitro experimental approach. Biochim Biophys Acta Gen Subj 1863:586-597.

De Petrocellis L, Ligresti A, Moriello AS, Allarà M, Bisogno T, Petrosino S, Stott CG, and Di Marzo V (2011) Effects of cannabinoids and cannabinoid-enriched Cannabis extracts on TRP channels and endocannabinoid metabolic enzymes. Br J Pharmacol 163:1479-1494.

De Petrocellis L, Orlando P, Moriello AS, Aviello G, Stott C, Izzo AA, and Di Marzo V (2012) Cannabinoid actions at TRPV channels: effects on TRPV3 and TRPV4 and their potential relevance to gastrointestinal inflammation. Acta Physiol (Oxf) 204: 255-266.

Díaz-Alonso J, Paraíso-Luna J, Navarrete C, Del Río C, Cantarero I, Palomares B, Aguareles J, Fernández-Ruiz J, Bellido ML, Pollastro F, et al. (2016) VCE-003.2, a novel cannabigerol derivative, enhances neuronal progenitor cell survival and alleviates symptomatology in murine models of Huntington's disease. Sci Rep 6 : 29789

Echeverry C, Prunell G, Narbondo C, de Medina VS, Nadal X, Reyes-Parada M, and Scorza C (2020) A Comparative in vitro study of the neuroprotective effect induced by cannabidiol, cannabigerol, and their respective acid forms: relevance of the 5-HT. Neurotox Res. doi: 10.1007/s12640-020-00277-y. [published ahead of print].

Ernsberger P, Giuliano R, Willette RN, and Reis DJ (1990) Role of imidazole receptors in the vasodepressor response to clonidine analogs in the rostral ventrolateral medulla. J Pharmacol Exp Ther 253:408-418.

Farha MA, El-Halfawy OM, Gale RT, MacNair CR, Carfrae LA, Zhang X, Jentsch NG, Magolan J, and Brown ED (2020) Uncovering the hidden antibiotic potential of cannabis. ACS Infect Dis 6:338-346.

Farrimond JA, Whalley BJ, and Williams CM (2012) Cannabinol and cannabidiol exert opposing effects on rat feeding patterns. Psychopharmacology (Berl) 223: 117-129.

Fellermeier M, Eisenreich W, Bacher A, and Zenk MH (2001) Biosynthesis of cannabinoids. Incorporation experiments with (13)C-labeled glucoses. Eur J Biochem 268:1596-1604.

Fellermeier M and Zenk MH (1998) Prenylation of olivetolate by a hemp transferase yields cannabigerolic acid, the precursor of tetrahydrocannabinol. FEBS Lett 427: $283-285$.

Fellous T, De Maio F, Kalkan H, Carannante B, Boccella S, Petrosino S, Maione S, Di Marzo V, and Iannotti FA (2020) Phytocannabinoids promote viability and functional adipogenesis of bone marrow-derived mesenchymal stem cells through different molecular targets. Biochem Pharmacol 175:113859. 
García C, Gómez-Cañas M, Burgaz S, Palomares B, Gómez-Gálvez Y, Palomo-Garo C, Campo S, Ferrer-Hernández J, Pavicic C, Navarrete C, et al. (2018) Benefits of VCE-003.2, a cannabigerol quinone derivative, against inflammation-driven neuronal deterioration in experimental Parkinson's disease: possible involvement of different binding sites at the PPAR y receptor. J Neuroinflammation 15:19.

Gertler R, Brown HC, Mitchell DH, and Silvius EN (2001) Dexmedetomidine: a novel sedative-analgesic agent. Proc Bayl Univ Med Cent 14:13-21.

Granja AG, Carrillo-Salinas F, Pagani A, Gómez-Cañas M, Negri R, Navarrete C, Mecha M, Mestre L, Fiebich BL, Cantarero I, et al. (2012) A cannabigerol quinone alleviates neuroinflammation in a chronic model of multiple sclerosis. J Neuroimmune Pharmacol 7:1002-1016.

Gugliandolo A, Silvestro S, Chiricosta L, Pollastro F, Bramanti P, and Mazzon E (2020) The transcriptomic analysis of NSC-34 motor neuron-like cells reveals that cannabigerol influences synaptic pathways: a comparative study with cannabidiol. Life (Basel) 10:227.

Gülck T and Møller BL (2020) Phytocannabinoids: origins and biosynthesis. Trends Plant Sci 25:985-1004.

Hunter JC, Fontana DJ, Hedley LR, Jasper JR, Lewis R, Link RE, Secchi R, Sutton J, and Eglen RM (1997) Assessment of the role of alpha2-adrenoceptor subtypes in the antinociceptive, sedative and hypothermic action of dexmedetomidine in transgenic mice. Br J Pharmacol 122:1339-1344.

Jones NA, Hill AJ, Smith I, Bevan SA, Williams CM, Whalley BJ, and Stephens GJ (2010) Cannabidiol displays antiepileptiform and antiseizure properties in vitro and in vivo. J Pharmacol Exp Ther 332:569-577.

Kinghorn A, Falk H, Gibbons S, and Kobayashi J (2017) Phytocannabinoids, Springer, Switzerland.

Kocis PT and Vrana KE (2020) Delta-9-Tetrahydrocannabinol and cannabidiol drugdrug interactions. Med Cannabis Cannabinoids 3:61-73.

Mammana S, Cavalli E, Gugliandolo A, Silvestro S, Pollastro F, Bramanti P, and Mazzon E (2019) Could the combination of two non-psychotropic cannabinoids counteract neuroinflammation? Effectiveness of cannabidiol associated with cannabigerol. Medicina (Kaunas) 55:747.

Molinoff PB (1984) Alpha- and beta-adrenergic receptor subtypes properties, distribution and regulation. Drugs 28 (Suppl 2):1-15.

Muller C, Morales P, and Reggio PH (2019) Cannabinoid ligands targeting TRP channels. Front Mol Neurosci 11:487.

Navarro G, Varani K, Lillo A, Vincenzi F, Rivas-Santisteban R, Raïch I, Reyes-Resina I, Ferreiro-Vera C, Borea PA, Sánchez de Medina V, et al. (2020) Pharmacological data of cannabidiol- and cannabigerol-type phytocannabinoids acting on cannabinoid $\mathrm{CB}_{1}, \mathrm{CB}_{2}$ and $\mathrm{CB}_{1} / \mathrm{CB}_{2}$ heteromer receptors. Pharmacol Res 159:104940.

Navarro G, Varani K, Reyes-Resina I, Sánchez de Medina V, Rivas-Santisteban R, Sánchez-Carnerero Callado C, Vincenzi F, Casano S, Ferreiro-Vera C, Canela EI, et al. (2018) Cannabigerol action at cannabinoid $\mathrm{CB}_{1}$ and $\mathrm{CB}_{2}$ receptors and at $\mathrm{CB}$ ${ }_{1}-\mathrm{CB}{ }_{2}$ heteroreceptor complexes. Front Pharmacol 9:632.

Nichols DE and Nichols CD (2008) Serotonin receptors. Chem Rev 108:1614-1641.

O'Sullivan SE (2016) An update on PPAR activation by cannabinoids. Br J Pharmacol 173:1899-1910

O'Sullivan SE and Kendall DA (2010) Cannabinoid activation of peroxisome proliferator-activated receptors: potential for modulation of inflammatory disease. Immunobiology 215:611-616.

Pagano E, Iannotti FA, Piscitelli F, Romano B, Lucariello G, Venneri T, Di Marzo V, Izzo AA, and Borrelli F (2020) Efficacy of combined therapy with fish oil and phytocannabinoids in murine intestinal inflammation. Phytother Res. DOI: 10.1002/ptr.6831 [published ahead of print].

Pavlovic R, Nenna G, Calvi L, Panseri S, Borgonovo G, Giupponi L, Cannazza G, and Giorgi A (2018) Quality traits of "cannabidiol oils": cannabinoids content, terpene fingerprint and oxidation stability of European commercially available preparations. Molecules 23:1230.

Pertwee RG (2008) The diverse CB1 and CB2 receptor pharmacology of three plant cannabinoids: delta9-tetrahydrocannabinol, cannabidiol and delta9-tetrahydrocannabivarin Br J Pharmacol 153:199-215.

Philipp M, Brede M, and Hein L (2002) Physiological significance of alpha(2)-adrenergic receptor subtype diversity: one receptor is not enough. Am J Physiol Regul Integr Comp Physiol 283:R287-R295.

Pinzi L, Lherbet C, Baltas M, Pellati F, and Rastelli G (2019) In silico repositioning of cannabigerol as a novel inhibitor of the enoyl Acyl Carrier Protein (ACP) reductase (InhA). Molecules 24:2567.
Pollastro F, Taglialatela-Scafati O, Allarà M, Muñoz E, Di Marzo V, De Petrocellis L, and Appendino G (2011) Bioactive prenylogous cannabinoid from fiber hemp (Cannabis sativa). J Nat Prod 74:2019-2022.

Raup-Konsavage W, Carkaci-Salli N, Greenland K, Gearhart RJ, and Vrana K (2020) Cannabidiol (CBD) oil does not display an entourage effect in reducing cancer cell viability in vitro. Med Cannabis Cannabinoids Available from: https://www.karger. com/Article/FullText/510256 Published ahead of print.

Rock EM, Bolognini D, Limebeer CL, Cascio MG, Anavi-Goffer S, Fletcher PJ, Mechoulam R, Pertwee RG, and Parker LA (2012) Cannabidiol, a non-psychotropic component of cannabis, attenuates vomiting and nausea-like behaviour via indirect agonism of 5-HT(1A) somatodendritic autoreceptors in the dorsal raphe nucleus. $\mathrm{Br}$ J Pharmacol 165:2620-2634.

Rock EM, Goodwin JM, Limebeer CL, Breuer A, Pertwee RG, Mechoulam R, and Parker LA (2011) Interaction between non-psychotropic cannabinoids in marihuana: effect of cannabigerol (CBG) on the anti-nausea or anti-emetic effects of cannabidiol (CBD) in rats and shrews. Psychopharmacology (Berl) 215:505-512.

Rodríguez-Cueto C, Santos-García I, García-Toscano L, Espejo-Porras F, Bellido M, Fernández-Ruiz J, Muñoz E, and de Lago E (2018) Neuroprotective effects of the cannabigerol quinone derivative VCE-003.2 in SOD1 G93A transgenic mice, an experimental model of amyotrophic lateral sclerosis. Biochem Pharmacol 157: 217-226.

Rojas PS and Fiedler JL (2016) What do we really know about 5-HT ${ }_{1 \mathrm{~A}}$ receptor signaling in neuronal cells? Front Cell Neurosci 10:272.

Rosenthaler S, Pöhn B, Kolmanz C, Huu CN, Krewenka C, Huber A, Kranner B, Rausch WD, and Moldzio R (2014) Differences in receptor binding affinity of several phytocannabinoids do not explain their effects on neural cell cultures. Neurotoxicol Teratol 46:49-56.

Russo EB (2016) Beyond cannabis: plants and the endocannabinoid system. Trends Pharmacol Sci 37:594-605.

Russo EB, Burnett A, Hall B, and Parker KK (2005) Agonistic properties of cannabidiol at 5-HT1a receptors. Neurochem Res 30:1037-1043.

Ryberg E, Larsson N, Sjögren S, Hjorth S, Hermansson NO, Leonova J, Elebring T, Nilsson K, Drmota T, and Greasley PJ (2007) The orphan receptor GPR55 is a novel cannabinoid receptor. Br J Pharmacol 152:1092-1101.

Ryz NR, Remillard DJ, and Russo EB (2017) Cannabis roots: a traditional therapy with future potential for treating inflammation and pain. Cannabis Cannabinoid Res 2:210-216.

Sacco LN (2014) Drug enforcement in the United States: history, policy, and trends Available from: https://fas.org/sgp/crs/misc/R43749.pdf, 7-5700, Congressional Research Service, Washington D.C.

Saunders C and Limbird LE (1999) Localization and trafficking of alpha2-adrenergic receptor subtypes in cells and tissues. Pharmacol Ther 84:193-205.

Shoyama Y, Yagi M, Nishioka I, and Yamauchi T (1975) Biosynthesis of cannabinoid acids. Phytochemistry 14:2189-2192.

Tudge L, Williams C, Cowen PJ, and McCabe C (2014) Neural effects of cannabinoid CB1 neutral antagonist tetrahydrocannabivarin on food reward and aversion in healthy volunteers. Int $J$ Neuropsychopharmacol 18:pyu094

Urasaki Y, Beaumont C, Workman M, Talbot JN, Hill DK, and Le TT (2020) Potency assessment of CBD oils by their effects on cell signaling pathways. Nutrients 12: 357.

Uys MM, Shahid M, and Harvey BH (2017) Therapeutic potential of selectively targeting the $\alpha_{2 \mathrm{C}}$-adrenoceptor in cognition, depression, and schizophrenia-new developments and future perspective. Front Psychiatry 8:144.

Valdeolivas S, Navarrete C, Cantarero I, Bellido ML, Muñoz E, and Sagredo O (2015) Neuroprotective properties of cannabigerol in Huntington's disease: studies in R6/2 mice and 3-nitropropionate-lesioned mice. Neurotherapeutics 12:185-199.

Volkow ND, Baler RD, Compton WM, and Weiss SR (2014) Adverse health effects of marijuana use. $N$ Engl J Med 370:2219-2227.

Wirth PW, Watson ES, ElSohly M, Turner CE, and Murphy JC (1980) Antiinflammatory properties of cannabichromene. Life Sci 26:1991-1995.

Address correspondence to: Kent E. Vrana, Penn State College of Medicine, 500 University Dr., Hershey, PA 17033. E-mail: kvrana@pennstatehealth.psu.edu 